\title{
Dietary Fiber and the Risk of Pancreatic Cancer
}

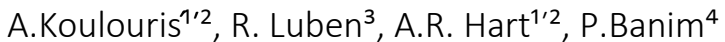

Dr Andreas I.Koulouris ${ }^{122}$, MSc, Academic Clinical Fellow.

Dr Robert Luben³ ${ }^{3}$ PhD, Head of Bioinformatics, EPIC Database Manager.

Dr Paul Banim4, MD, Consultant in Gastroenterology.

Professor Andrew R.Hart'², PhD, Professor in Gastroenterology.

\section{Author Affiliations}

${ }^{1}$ Norwich Medical School, University of East Anglia, Norwich, United Kingdom.

${ }^{2}$ Department of Gastroenterology, Norfolk and Norwich University Hospital, Norwich, United Kingdom.

${ }^{3}$ Institute of Public Health, University of Cambridge, United Kingdom.

${ }^{4}$ Department of Gastroenterology, James Paget University Hospital, Great Yarmouth, United Kingdom.

Corresponding author: Andreas Koulouris, a.koulouris@uea.ac.uk, Norwich Medical School, Bob Champion Building, University of East Anglia, Colney Lane, NR4 7UY, tel no: 00441603593611

Key words: Pancreatic cancer, nutrition, diet, fiber, etiology.

Conflicts of Interest and Source of Funding: All authors have completed the ICMJE uniform disclosure form at www.icmje.org/update.html and declare: no support from any organisation for the submitted work; no financial relationships with any organisations that might have an interest in the submitted work; no other relationships or activities that could appear to have influenced the submitted work. 


\section{Abstract}

Objectives: High dietary fiber may protect against pancreatic adenocarcinoma (PDA). We investigated associations between fiber intake and the risk of PDA using, for first time, 7- day food diaries (7-DFDs).

Methods: Participants in the European Prospective Investigation Cohort - Norfolk completed 7-DFDs at recruitment. The cohort was followed up for 17 years to identify those who developed PDA. Participants were divided in quintiles of fiber intake and hazard ratios (HR) estimated with their 95\% Confidence Intervals $(\mathrm{Cl})$. Fiber tested for effect modification of high red and processed meat intakes and smoking and the risk of PDA.

Results: No significant associations for any quintiles of intake $\left(H R \mathrm{O}_{5} \mathrm{Vs}_{\mathrm{1}}, 1.08 ; 95 \% \mathrm{Cl}, 0.56-2.08\right)$ were detected with no trend across quintiles. A high fiber diet modified positive associations between red and processed meats with the development of PDA (HR trend, $0.89 ; 95 \% \mathrm{Cl}, 0.47-1.69$ and 1.02; 95\% $\mathrm{Cl}, 0.55-1.88$ respectively), but not those with lower fiber intakes. Fiber intake did not modify the risk of PDA in past and current smokers.

Conclusion: The findings do not suggest fiber protects against PDA, although it may decrease potential deleterious effects of meats. 


\section{Introduction}

Pancreatic ductal adenocarcinoma (PDA) is the fifth commonest cancer worldwide, with 178,161 new cases diagnosed in $2012^{(1)}$. Even with potentially curative surgery the 5 -year survival is still only $21 \%{ }^{(2)}$. Understanding the aetiology of PDA is therefore important to inform public health recommendations to reduce its incidence. Components of habitual diet, including fiber, are plausible etiological risk factors to investigate which may explain the changes in PDA incidence over time and across different geographical regions ${ }^{(1)}$.

A high dietary fiber intake may hypothetically help prevent PDA by influencing: metabolic processes, reducing adiposity, oxidative stress and the absorption of dietary carcinogens. The former are: hyperinsulinaemia, hyperglycaemia, and fat and carbohydrate metabolism, which are all positively associated with the risk of developing PDA ${ }^{(3)}$. Fiber displaces carbohydrates from their absorption sites on the small intestinal mucosa leading to lower glucose and insulin levels post-prandially, which protects against developing type two diabetes a positive risk factor for pancreatic cancer ${ }^{(4)}$. Excess insulin is also mitogenic as it activates intracellular pathways, namely PI3K and mTOR signaling which is particularly relevant to PDA as insulin is secreted by the pancreas ${ }^{(5)}$. Individuals in the highest quintile of hyperglycaemia and hyperinsulinaemia have double the risk of PDA ${ }^{(6)}$. Importantly, a high fiber intake is associated with a reduction in $\mathrm{HbA1C}$ and serum insulin levels ${ }^{(7)}$. Hyperlipidaemia is a further positive risk factor for PDA which a high fiber diet may reduce as it promotes faster bile excretion, leading to lower serum LDL cholesterol ${ }^{(8)}$. Furthermore, excess adiposity is strongly associated with PDA, with a $19 \%$ increase for every rise in unit of BMI ${ }^{(9)}$ - (11). Fiber protects against adiposity by promoting early satiety and those with the highest fiber intakes were less likely to be obese compared to those with the lowest ones (Relative Risk [RR], 0.70; $95 \% \mathrm{Cl}, 0.62-0.78)^{(12)}$. Additionally, fiber-rich foods contain other components which have antioxidant and anti-proliferative properties including: vitamins $\mathrm{C}, \mathrm{E}$ and selenium, which inactivate free radicals responsible for DNA damage (13), (14), (15). Finally, dietary fiber may decrease the available carcinogen load delivered to the pancreas by increasing stool bulk. The later firstly dilutes stool concentrations of carcinogens and secondly, activates intestinal mechanoreceptors leading to quicker bowel transit times. These dilutional effects and the decrease in bowel transit times may be beneficial against the potential carcinogenic effect of high meat consumption. Cooking meats at high temperatures produces potential carcinogens, namely heterocyclic amines and polyaromatic hydrocarbons, and also meat preservation forms hazardous nitrosamimes ${ }^{(16),(17)}$. We have previously 
documented in this cohort positive associations with higher red and processed meat intakes in participants younger than 60 years at recruitment ${ }^{(18) .}$

The plausible biological mechanisms for how an increased dietary fiber intake may prevent PDA need to be supported by etiological epidemiological studies showing that people with higher fiber consumption are at a decreased risk of developing PDA. However, the results from the current epidemiological studies are inconsistent with a meta-analysis of 13 case-control studies reporting an inverse association (summary odd ratio $[\mathrm{OR}], 0.54 ; 95 \% \mathrm{Cl}, 0.44-0.67)^{(19)}$, but the only prospective cohort investigation documenting a null result (hazard ratio $[\mathrm{HR}], 1.01 ; 95 \% \mathrm{Cl}, 0.59-1.74, P=0.90$ ) ${ }^{(6)}$. However, all the retrospective studies have inherent selection and recall biases which may lead to spurious inverse associations. Furthermore, all these epidemiological studies used either: food frequency questionnaires or 48-hour recall diaries with a 7-day menu book to measure fiber intakes which have measurement errors for habitual diet. To the best of our knowledge, no etiological studies have used 7-day food diaries (7- DFDs), the most accurate pragmatic dietary assessment in large epidemiological studies. The accuracy of 7-DFDs, compared to 24 hour recalls and FFQs in measuring fiber intakes was reported in a validation study where fiber consumption for each of these three methods was compared with a 16-day weighed food records as the standard ${ }^{(20)}$. The reported Spearman correlation coefficients were highest for the 7-DFDs: (0.73) compared to 0.58 for 24 hour recalls and 0.55 for FFQs $(P<0.0001)^{(20)}$.

The aim of this epidemiological study was to investigate if there were inverse associations between dietary fiber intake and the development of PDA, using for first time 7-DFDs, in the European Prospective Investigation In to Cancer (EPIC)-Norfolk, to help clarify the inconsistencies in the current literature. To provide supportive mechanistic information for a role of both high red and processed meat intakes with pancreatic carcinogenesis we investigated if the intakes of these two food groups were modified by high and low fiber intakes. The findings would help inform whether dietary fiber intakes should be measured in future etiological studies of PDA, and possibly offer a method to prevent this highly aggressive malignancy.

\section{Methods and materials}

The EPIC-Norfolk study consists of 23,658 men and women, aged 40-74 years old, who were recruited between the years 1993-1997. Participants were registered in 35 general practices in: rural, suburban and urban areas in the county of Norfolk, UK. At recruitment participants completed questionnaires on their: demographics, medical history, drug and smoking history and diet and attended health checks where their height and weight were measured to calculate body mass index $\left(\mathrm{BMI} \mathrm{kg} / \mathrm{m}^{2}\right)$. The first day 
of the 7-DFDs was completed with the assistance of the research nurse at the health check, which was a recall of participants' dietary intake over the previous day. Participants then completed the subsequent 6 days of their diaries independently at home. Participants recorded the content and frequency of meals eaten, food types, portion sizes, brands, cooking methods and recipes at eight different stated meal and snack times each day. Portion sizes were estimated using standard units of measurement or photographs of different meal sizes included in the diary. The completed diaries were then returned to the research center where trained coders reviewed them and matched each food item with one of 11,000 food items and 55,000 portion sizes included in a software program called: DINER (Data Into Nutrients for Epidemiological Research) ${ }^{(21)}$. DINER contains nutrient information based on the: United Kingdom Food Composition Database, Royal Society of Chemistry and food manufacturers' databases. DINER facilitates the conversion of participant-reported free text into structured nutrient data including fiber. The program also checked for duplications or unexpected food values, such as excess portion sizes, which were then reviewed by the nutritionists. Each diary required approximately 2.5 hours to code and participants recorded an average of total 220 food and drink items consumed in the week. An example of the detail of DINER is that 337 different types and brands of cereal breakfast, an important source of dietary fiber were contained in DINER.

After recruitment participants were followed up until the year 2010, and those who developed PDA were identified by matching the EPIC-Norfolk database with: firstly the Norfolk District Health Authority records of hospital admissions and secondly the Eastern Cancer Registry and Information Centre (ECRIC). The medical notes were then reviewed by a medical gastroenterologist to ensure that participants had a confirmed diagnosis of PDA based on both the appropriate symptoms and radiological investigations. Participants with an uncertain clinical diagnosis of PDA and those diagnosed with PDA diagnosed within 12 months after enrolment, were excluded. The latter was to ensure the nutritional information was truly representative of their diet before the development of symptoms. The EPIC-Norfolk study was approved by the Norwich District Health Authority ethics committee. All the participants gave permissions for their medical notes to be reviewed for relevant clinical information.

A case-cohort analysis was conducted, comparing those with PDA and a subgroup of 3970 randomly selected controls from the entire EPIC-Norfolk database. The baseline characteristics between no-cases participants and participants with PDA were tested for differences using: $t$-test for continuous variables, the Mann-Whitney test for non-parametric variables and the $x^{2}$-test for dichotomous ones. Total fiber intake was divided into quintiles across the distribution of the whole cohort and Cox proportional hazard regression calculated $\mathrm{HRs}$ and $95 \% \mathrm{Cls}$ using the lowest quintile of fiber intake as the reference. The HRs were adjusted for the covariates of: age at recruitment, sex, diabetes mellitus, total energy intake (quintiles) and smoking status (never, former or current). Trends across quintiles were 
calculated, using the median value of each category as a continuous variable. In a further analysis, fiber intake was analysed as a dichotomous variable based on the mean daily fiber intake for the UK populations namely: above and below 20 grams/day ${ }^{(22)}$. A further analysis of the risk of PDA was conducted according to fruit and vegetable fiber sources, as the enzymatic resistance, particle size, bulk volume, porosity and solubility of the fiber varies depending on the source which influence its biochemical behavior ${ }^{(23)}$.

We conducted several sensitivity analyses related to meat intakes and smoking status. As we have previously documented both red and processed meats are associated with an increased risk of PDA in individuals younger than $60^{(18)}$ we assessed if there was effect modification for these meats according to high and low fiber intakes. Cooking meat at high temperatures or processing meat, both result in production of carcinogens, namely: heterocyclic amines, polyaromatic hydrocarbons and nitrosamines (16), (17). As the incidence of PDA peaks at the seventh decade of life ${ }^{(1)}$, exposure to these carcinogens prior to this time may contribute to pancreatic epithelial damage. This consists of, mutagenesis and progression from normal pancreatic ductal epithelium to epithelial metaplasia and later to pancreatic intraepithelial neoplasia (PanIN), the immediate PDA precursor ${ }^{(24)}$. We hypothesized that a high fiber diet might reduce this risk by decreasing intestinal transit times and reducing carcinogen absorption. To evaluate if there was effect modification of the associations for meat we investigated the trends of the risk for PDA across the quintiles of red and processed meats, for individuals younger than 60 years at recruitment consuming, firstly, below and, secondly, above the recommended daily fiber intakes. Finally, sensitivity analyses for fiber intakes were conducted for smokers (combined current and former) versus non-smokers, as antioxidants in fiber containing foods, may oppose the carcinogenic action of pro-oxidants in cigarettes. The study was approved by the Norwich Ethics Committee and all participants gave consent for their notes to be reviewed.

\section{Results}

In the 23,658 participants in EPIC- Norfolk, 88 (0.37\%) were diagnosed with PDA, during 17 years follow up, with a mean time from recruitment to diagnosis of 8.4 years (Standard Deviation, $[S D]=3.9$ ) (table 1). Cases were approximately five years older at recruitment than non-cases. In those participants who developed PDA, 74\% had metastatic disease at diagnosis and 35\% had cancer confirmed by histology or cytology Pancreatic surgery was performed in $8 \%$, with $35 \%$ receiving chemotherapy and the remainder (57\%) were referred for palliative supportive treatments. In cases where histology was not obtained, all had symptoms of PDA and at least two different radiological modalities confirming the diagnosis. The sample of non-cases had comparable demographics to those in the total cohort (mean age 59.3 vs 59.0 years, men $43.8 \%$ vs $45.5 \%)$. Only 39 (1.0\%) of the 3,790 non-cases and 2 (3.3\%) of 88 
cases had missing information on smoking status. Cases and non-cases had similar mean daily total fiber intakes (table 1). Only $16 \%(n=615)$ of both groups ate more than the average daily fiber intake of $20 \mathrm{~g}$ of the British population ${ }^{(22)}$. No cases and $1.5 \%(n=61)$ of non-cases, were consuming above the recommended daily fiber intakes of $30 \mathrm{~g}$ for adults, as recommended by the British Nutrition Foundation (22).

In the regression analysis, the development of PDA was not associated with the daily fiber intake for any of the higher quintiles compared to the lowest one (e.g. $H R \mathrm{O}_{5} \mathrm{Vs}_{\mathrm{N}} \mathrm{Q}_{1}, 1.08 ; 95 \%, 0.56-2.08$ ) (table 2). There were no trends across quintiles and the results were similar when adjusted for the additional co-variates in the multivariable analyses. There were no associations in a binary analysis for participants consuming more than 20 grams fiber per day (mean fiber daily consumption of the UK population)(HR, $1.12 ; 95 \% \mathrm{Cl} ; 0.62-2.05, P=0.69]$. There were no associations between PDA and the daily consumption of fiber from vegetables for each of the higher quintiles compared to the lowest or for trend across quintiles ( $H R$ trend, 1.03; $95 \% \mathrm{Cl}, 0.89-1.20, P=0.60$ ) and similarly for fiber from fruits (HR trend, 0.99; $95 \% \mathrm{Cl}, 0.99-1.00, P=0.90$ ) (Data on individual quantities not shown). For meat intakes, there was a statistically significant increase in the risk for trend of PDA across quintiles of both red and processed meat intakes for individuals in the low fiber intake below the age of recruitment of 60 years (HR trend red meat, 1.45; $95 \% \mathrm{Cl}, 1.05-2.01$, HR trend processed meat, 1.46; 95\% Cl, 1.06-2.02) (table 3). For participants with the higher fiber intakes, no associations were found for the trends across quintiles of either higher red or processed meat intakes. Similarly, there was no effect modification according to smoking status for total fiber intake (data not shown).

\section{Discussion}

The main finding of this etiological study was that higher dietary fiber intakes were not associated with the risk of developing PDA. These null associations were consistent for fiber intakes according to either: quintiles of fiber, the recommended daily fiber intake, fiber sources namely: vegetables and fruits, or according to smoking status. These null findings are despite several plausible mechanisms for how fiber may protect against the development of PDA ${ }^{(11),(10)}$. Dietary fiber displaces carbohydrates from absorption sites in the small intestine, reducing their uptake and consequently decreasing insulin secretion ${ }^{(7) .}$ Hyperinsulinaemia is positively associated with carcinogenesis, as insulin stimulates the proliferation of pancreatic cells through activation of the IGF (Insulin Growth Factor) receptor and reduction of IGF-binding protein ${ }^{(11),(10)}$. Similarly, dietary fiber decreases lipid absorption and promotes its excretion in the feces ${ }^{(25)}$. Lipids are calorie dense nutrients with known pro-inflammatory properties and are strongly associated with: obesity, diabetes and the metabolic syndrome. Those with high lipid 
intakes are at an increased risk of developing PDA compared to those with low ones [summary relative risk, 1.08; 95\% Cl, 1.04-1.13] ${ }^{(26)}$. Furthermore, fiber induces early satiety and prevents obesity, another positive risk factor for PDA (summary relative risk, 1.14; $95 \% \mathrm{Cl}, 1.07-1.22)$ per unit $\left(5 \mathrm{~kg} / \mathrm{m}^{2}\right)^{(27)}$. Smaller effect sizes for fiber may exist but this study did not have sufficient statistical power to detect these. Also, assuming that if fiber has a protective effect with intakes above the recommended level, it would have been difficult for our study to detect this difference when $84 \%$ of participants consumed below this amount.

Our study reported that the positive associations with higher red and processed meat intakes, which we have previously documented in this cohort ${ }^{(18)}$, persisted in participants consuming less than $20 \mathrm{~g}$ /day of fiber in those recruited younger than 60 years. However, there was some evidence that this association disappeared in those eating higher amounts of fiber, although the estimates lacked precision due to the small number of cases. Cooking red meat at higher temperatures releases heterocyclic amines (HCAs) and polyaromatic hydrocarbons (PAHs) and processed meat adds nitrosamines ${ }^{(16),(17)}$. These carcinogens may induce the pancreatic ductal cells to undergo squamous metaplasia, then progress to pancreatic intraepithelial neoplasias (PanINs) and eventually to PDA. The incidence of PDA rises dramatically after the age of 60 years and it is thought that metaplasia and PanIN changes precede this by 10 years ${ }^{(24)}$. Individuals younger than 60 years who consume high amounts of red and processed meats may therefore be more susceptible to pancreatic carcinogenesis. Higher fiber intakes may dilute the concentrations of potential meat carcinogens in food and also decrease intestinal transit times, reducing their absorption. We reported that individuals with: high red and processed meat intakes and simultaneously low fiber daily intakes, had a $45 \%$ and $46 \%$ higher risks for developing PDA compared to no increase in risk with higher meat intakes in those eating more fiber. The cohort will be continued to be followed up to identify more cases of PDA to increase the statistical precision of these estimates with higher fiber intakes to confirm if these are indeed null associations.

Our study has several methodological strengths which supports the validity of these findings. These include the accuracy of the 7-DFD for recording habitual diet and the prospective study design which minimizes both selection and recall biases, which are inherent in retrospective case-control studies. The 7-DFDs are more accurate than both FFQs and 24 hour recall diaries for measuring diet (20). Estimates of fiber intake obtained by these three methods are assessed against the standard of 16-day weighed records and the 7-DFD had the highest correlation. The 7-DFDs may be more accurate as all foods and drink intakes are reported when actually consumed, and later interpreted using a comprehensive nutrient database. Furthermore, our findings are generalizable as participants' demographic and clinical characteristics were comparable with those of patients with PDA worldwide ${ }^{(28)}$. Also we performed a binary analysis according to the average daily fiber intake in the UK 
population to assess representativeness. Misclassification bias was avoided as all the medical notes of patients were reviewed by a medical gastroenterologist to confirm the diagnoses. Moreover, we used two separate sources to identify cases of PDA namely: hospital records and the regional cancer registry, to minimize follow-up bias. Follow-up bias will be low as after 20 years of commencement of the EPIC study, $96 \%$ of participants are still resident in the county of Norfolk. Finally, the hazards ratios were adjusted for known co-variates for PDA namely: diabetes, smoking status and total calorie intake. The reason the number of cases of PDA with histological confirmation was relatively small, was that most were diagnosed in the years before endoscopic ultrasound guided FNA was generally available in many UK hospitals. However, the characteristics of cases with and without histology were similar including the demographics and proportion with metastatic disease. A weakness of our study was the relatively small number of cases which could have obscured any small associations of fiber with PDA being detected. However, the cohort is continuing to be monitored to accrue more cases to increase the statistical precision of the associations. Finally, the duration of follow up was 17 years, during which participants may have changed their diet, introducing measurement error for diet. However, previous studies have reported relatively small changes in diet measured by food frequency questionnaires completed annually for 5 years ${ }^{(29)}$. Although some dietary changes may occur, these are probably relatively small and unlikely to result in a participant being allocated into a different quintile of fiber intake.

Our findings of a null association with fiber intake is consistent with that reported in the only other prospective cohort study investigating this association. Here in 27.111 Finish male smokers, with their habitual diet measured using FFQs, 163 (0.06\%) developed PDA during 13-years of follow up. The mean fiber intake was $24 \mathrm{~g} /$ day, which was higher than in our cohort (14.2 g/day). The authors also reported no tend across quintiles of fiber intakes with the risk of PDA $(H R, 1.01 ; 95 \% \mathrm{Cl}, 0.59-1.74, P=0.90)^{(6)}$. However, a meta-analysis of 13 case-control studies reported a summary odds ratio for the highest versus the lowest category $0.54(95 \% \mathrm{Cl}, 0.44-0.67, P=0.043)^{(19)}$. Of these, 12 reported an odd ratios below 1 (11 of them statistically significant), and the other an odd ratio 1.44 ( $95 \% \mathrm{Cl}, 0.70-2.95)$ for men and $0.28(95 \% \mathrm{Cl} 0.12-0.57)$ for women ${ }^{(30)}$. Six studies used self-reported FFQs and 7 proxy respondent FFQs, including those from partners, spouses, carers and relatives, recording retrospectively dietary intake 1-5 years before the diagnosis of PDA was made. As this dietary information was collected after the diagnosis of PDA, and by proxy respondents, the fiber intake is likely to be subject to recall bias for fiber intake. Participants with PDA may not be able to accurately recall their pre-symptomatic intake and report their current consumption which may be reduced by their symptoms and therefore not reflect that involved in aetiology. This recall bias may lead to spurious 
inverse associations with fiber reported in case-control work. Furthermore, none of the previous studies assessed for effect of modification of fiber on red and processed meat intake.

In summary, this study does not support a role for high fiber intakes protecting against the development of PDA although there may be a benefit in those with high meat intakes. Further prospective cohort studies are now required to investigate if these findings are consistent. If so, the risk of PDA may be reduced by recommending particular dietary patterns such as those with high meat intakes also have a high fiber intake.

\section{Acknowledgements.}

EPIC participants who participated in the study. The Norfolk-based Big C cancer charity who provided specific funding for this work, and the Medical Research Council and Cancer Research UK who supported the infrastructure of the main work.

\section{References}

1. Globocan. Estimated cancer incidence, mortality and prevalence worldwide in 2012. World Health Organisation (WHO) website. October 9, 2014. Available at: http://globocan.iarc.fr/Pages/fact_sheets_population.aspx. Accessed July 15, 2018.

2. McPhail, Oehler, Sreeharan et al. National Cancer Intelligence Network Cancer by Deprivation in England Incidence, 1996-2010 Mortality, 1997-2011. Cancer Research UK website. May 2014. Available at: http://www.cancerresearchuk.org/health-professional/cancer-statistics/statistics-bycancer-type/pancreatic-cancer/survival. Acessed: July 16, 2018.

3. Maisonneuve, Lowenfels, et al. Risk factors for pancreatic cancer: a summary review of metaanalytical studies. Int J Epidemiol. 2015;44: 186-198.

4. Larsson, Permert, Håkansson, et al. Overall obesity, abdominal adiposity, diabetes and cigarette smoking in relation to the risk of pancreatic cancer in two Swedish population-based cohorts. Br J Cancer. 2005;93:1310-1305.

5. Trajkovic-Arsic, Kalideris, Siveke, et al.The role of insulin and IGF system in pancreatic cancer. J Mol Endocrinol. 2013;50:67-74.

6. Stolzenberg-Solomon, Graubard, Chari, et al. Insulin, glucose, insulin resistance and pancreatic cancer in male smokers. JAMA. 2005;294:2872-2878.

7. Silva, Kramer, de Almeida, et al. Fiber intake and glycemic control in patients with type 2 diabetes mellitus: a systematic review with meta-analysis of randomized controlled trials. Nutr Rev. 2013;71:790-801.

8. Wang, Wang, Zhai, et al. Association of cholesterol with risk of pancreatic cancer: A meta-analysis. World J Gastroenterol. 2015 Mar 28; 21: 3711-3719.

9. Berrington, Sweetland, Spencer, et al. A meta-analysis of obesity and the risk of pancreatic cancer. Br J Cancer. 2003; 89: 519-523. 
10. Wolpin, Michaud, Giovannucci, et al. Circulating insulin-like growth factor binding protein-1 and the risk of pancreatic cancer. Cancer Res. 2007;67:7923-7928.

11. Fisher, Boros, Schirmer, et al. Insulin promotes pancreatic cancer: evidence for endocrine influence on exocrine pancreatic tumors. J Surg Res. 1996;63:310-313.

12. Anderson, Richard, Davis, et al. Health benefits of dietary fiber. Nutr Rev. 2009;67:188-205

13. Wintergerst, Maggini, Hornig, et al. Immune-enhancing role of vitamin $\mathrm{C}$ and zinc and effect on clinical conditions. Ann Nutr Metab. 2006;50:85-94.

14. Meydani, Meydani, Blumberg, et al. Vitamin E supplementation and in vivo immune response in healthy elderly subjects. A randomized controlled trial. JAMA. 1997;277:1380-6

15. Bao, Michaud, Spiegelman, Albanes, et al. Folate Intake and Risk of Pancreatic Cancer: Pooled Analysis of Prospective Cohort. J Natl Cancer Inst. 2011;103:1840-50.

16. Yoshimoto, Tsutsumi, Iki, et al. Carcinogenicity of heterocyclic amines for the pancreatic duct epithelium in hamsters. Cancer Lett. 1999;143:235-239.

17. Dissin, Mills, Mains, et al. Experimental Induction of Pancreatic Adenocarcinoma in Rats. J Natl Cancer Inst. 1975;55:857-864.

18. Beaney, Banim, Luben, et al. Higher Meat Intake Is Positively Associated With Higher Risk of Developing Pancreatic Cancer in an Age-Dependent Manner and Are Modified by Plasma Antioxidants: A Prospective Cohort Study (EPIC-Norfolk) Using Data From Food Diaries. Pancreas. 2017;46:672-678.

19. Wang, Qiao, Wang, et al. Dietary fiber intake and pancreatic cancer risk: A meta-analysis of epidemiologic studies. Sci Rep. 2015;5:10834.

20. Bingham, Gill, Welch, et al. Validation of dietary assessment methods in the UK arm of EPIC using weighed records, and 24-hour urinary nitrogen and potassium and serum vitamin $\mathrm{C}$ and carotenoids as biomarkers. Int J Epidemiol. 1997;26:137-151.

21. Welch, McTaggart, Mulligan, et al. DINER (Data Into Nutrients for Epidemiological Research) - a new data-entry program for nutritional analysis in the EPIC-Norfolk cohort and the 7-day diary method. Public Health Nutr. 2001;4:1253-1265

22. British Nutrition Foundation. Dietary Fibre. Oct 2012. Available at: https://www.nutrition.org.uk/healthyliving/basics/fibre.html. Accessed July 15, 2018.

23. Dhingra, Michael, Rajput, et al. Dietary fibre in foods: a review. J Food Sci Technol. 2012; 49: 255266.

24. Hruban, Maitra, Goggins, et al. Update on Pancreatic Intraepithelial Neoplasia. Int J Clin Exp Pathol. 2008; 1: 306-316.

25. Kritchevsky. Dietary fibre and lipid metabolism. Int J Obes. 1987;11:33-43.

26. Chen, Qin, Wang, et al. Association between cholesterol intake and pancreatic cancer risk: Evidence from a meta-analysis. Sci Rep. 2015; 5: 8243.

27. Preziosia, Oben, Fusai, et al. Obesity and pancreatic cancer. Mol Carcinog. 2012; 51: 53-63.

28. Parkin, Bray , Ferlay, et al. Global cancer statistics. CA Cancer J Clin. 2005;55:74-108. 
29. Goldbohn, van 't Veer, van den Brandt, et al. Reproducibility of a food frequency questionnaire and stability of dietary habits determined from five annually repeated measurements. Eur J Clin Nutr. 1995;49:420-429.

30. Lyon, Slattery, Mahoney, Robinson, et al. Dietary intake as a risk factor for cancer of the exocrine pancreas. Cancer Epidemiol Biomarkers Prev. 1993;2:513-518. 